\title{
Editorial
}

\section{Communications and Networking for Mobile Sink in Wireless Sensor Networks}

\author{
Ki-Il Kim ${ }^{D},{ }^{1}$ Shuhui Yang, ${ }^{2}$ and Euisin Lee $^{3}$ \\ ${ }^{1}$ Department of Computer Science and Engineering, Chungnam National University, Daejeon 34134, Republic of Korea \\ ${ }^{2}$ Department of Computer Science, Purdue University Northwest, Hammond, IN 46323, USA \\ ${ }^{3}$ School of Information and Communication Engineering, Chungbuk National University, Cheongju, Republic of Korea
}

Correspondence should be addressed to Ki-Il Kim; kikim@cnu.ac.kr

Received 19 July 2021; Accepted 19 July 2021; Published 8 November 2021

Copyright (C $2021 \mathrm{Ki}-\mathrm{Il}$ Kim et al. This is an open access article distributed under the Creative Commons Attribution License, which permits unrestricted use, distribution, and reproduction in any medium, provided the original work is properly cited.

Wireless sensor networks (WSNs) have opened many new possibilities for emerging applications in event tracking and surveillance. However, when compared to the great research interest in this topic, only a limited number of frameworks have been deployed in the real world due to multihop and long hop delivery over many constraints on both nodes and networks. Currently, mobile sink nodes can be a feasible solution for data collection in an energy-efficient way to reduce the total energy consumption with a small number of hops. Unlike static sink approaches, the mobile sink approach requires a new paradigm to collect data such that the sensed data is correctly delivered to mobile nodes. In this situation, a packet may be delivered to a previous location, or a node needs to keep a packet until a mobile sink moves to a nearby place. In addition to these cases, the movement of mobile sink nodes may lead to many research challenges caused by their frequent path changes.

Despite these challenges, recently, there has been a large amount of research introducing mobile robots or unmanned aerial vehicles as mobile sinks. With the help of these systems, many research challenges can be simplified-however, the energy efficiency problem with respect to their communications remains unsolved. Hence, there is a tremendous need for researchers and engineers to have a comprehensive knowledge of the latest advances in mobile sink technology. Based on this demand, this special issue contributes to the advances in open technical problems and challenges in communication and networking for mobile sink in WSN, presenting original research articles that tackle the problem of efficient integration of novel solutions with existing mobile sink technologies.

While considering our objectives, the editors believe that this special issue provides a collection of articles on networking techniques in mobile sinks. We have selected seven valuable papers by evaluating several aspects such as relevance to the special issue and novelty of solutions. The topic of these papers is roughly categorized into the following areas: clustering, routing, OFDM, data distribution, and development of testbed.

In the paper entitled "Optimal Clustering in Wireless Sensor Networks for the Internet of Things Based on Memetic Algorithm: memeWSN," M. Ahmad et al. proposed a memetic algorithm (MemA) to decrease the probability of early convergence by utilizing local exploration techniques. In the proposed scheme, a cluster header in the cluster header set is dynamically selected as early as possible to reduce the convergence time. The proposed technique outperforms the existing scheme in the aspects of control message overhead, cluster count, reconstruction rate, and cluster lifetime. J. Park et al. presented a clustering as well as trajectory optimization scheme to improve energy efficiency in the second paper. The authors propose an iterative algorithm to minimize the amount of energy consumed by components of wireless sensor networks. The proposed scheme takes into account the density of sensors and residual battery of sensors when deciding a suitable number of clusters and cluster headers in the first phase. In the phase, the trajectory is optimized by formulating 
the suitable trajectories of multiple mobile sinks to minimize the amount of energy consumed by mobile sinks.

In addition to the clustering scheme, C. Kim et al. addressed a multipath routing protocol for mobile sinks. The proposed protocol dynamically constructs multipaths along the moving path of a sink with the help of agents. In addition, path shortening and path extending schemes are applied to reduce the impact of the multipath reconstruction interval and neighbor list update interval. Furthermore, S. Li and S. Kang presented new signal constellation pairs for mapping active subcarriers of the zero-padded trimode orthogonal frequency division multiplexing with index modulation (ZTM-OFDM-IM) systems and evaluated the performance in bit error rate.

The paper is about sensor clouds system, entitled "OEDDBOS: An Efficient Data Distributing Strategy with Energy Saving in Sensor-Cloud Systems." The authors addressed energy consumption for data dissemination issues and presented data distribution with delay demand. To achieve the research objectives, continuous monitoring for the variation of the channel quality as well as the decision for stopping time is introduced to maximize the expected reward of energy efficiency. The final paper is for a testbed system in Internet of Things (IoT). In this work, the authors present an IoT testbed system which can be used to run the experimentation of diverse IoT technologies. The system consists of a set of robust hardware components and software modules. The evaluation results for Bluetooth Low Energy (BLE), Zigbee, and 6LoWPAN technologies have been given. The last paper is for another deployment issued, entitled "Research on the Difficulty of Mobile Node Deployment's Self-play in Wireless Ad Hoc Networks Based on Deep Reinforcement Learning." H. Wang et al. addressed the fast convergence of deep reinforcement learning for node deployment in wireless networks. Dynamic updating learning rate and the method of selecting the latest model to generate sample data are proposed for AlphaZero algorithm.

\section{Conflicts of Interest}

I declare that none of the guest editors have a conflict of interest.

\section{Acknowledgments}

As guest editors, we appreciate all authors, reviewers, and editorial members for their invaluable contribution. Without their hard work and dedication, it would not have been possible to select these high-qualified papers within the given time limit of this special issue.

Ki-Il Kim

Euisin Lee

Shuhui Yang 